Comptes Rendus Acad. Sci. Paris

Analyse Mathematique / Mathematical Analysis

Rubrique secondaire: Analyse Harmonique / Harmonic Analysis

Titre français: Un raffinement du théorème de Erdős-Turán sur la distribution des zéros de polynômes

\title{
AN IMPROVEMENT OF THE ERDÖS-TURÁN THEOREM ON THE DISTRIBUTION OF ZEROS OF POLYNOMIALS
}

\author{
Tamás Erdélyi \\ Department of Mathematics, Texas A\&M University \\ College Station, Texas 77843, USA \\ E-mail: terdelyi@math.tamu.edu
}

\begin{abstract}
We prove a subtle "one-sided" improvement of a classical result of $\mathrm{P}$. Erdős and P. Turán on the distribution of zeros of polynomials. The proof of this improvement is quite short and rather elementary. Nevertheless it allows us to obtain a beautiful recent result of V. Totik and P. Varjú as a simple corollary, and in a somewhat stronger form, without any use of a potential theoretic machinery. Namely, if the modulus of a monic polynomial $P$ of degree $n$ (with complex coefficients) on the unit circle of the complex plane is at most $1+o(1)$ uniformly, then the multiplicity of each zero of $P$ on the unit circle is $o\left(n^{1 / 2}\right)$. Our approach is based on the interesting observation that the Erdős-Turán Theorem improves itself.
\end{abstract}

Résumé: Nous prouvons un raffinement délicat d'un résultat classique de P. Erdős et P. Turán sur la distribution des zéros de polynômes. Bien que notre preuve soit brève et plutôt élémentaire, elle nous permet d'obtenir comme corollaire et sans recourir à la théorie du potentiel, une amélioration d'un résultat récent et élégant de V. Totik et P. Varjú: si le module sur le cercle unité du plan complexe d'un polynôme $P$ monique, de degré $n$ et à coefficients complexes est uniformément au plus $1+o(1)$, alors la multiplicité de chaque zéro de $P$ sur le cercle unité est $o\left(n^{1 / 2}\right)$. Notre approche repose sur l'observation, à notre avis intéressante, que le théorème de Erdős-Turán puisse en quelque sorte s'auto-raffiner.

\section{INTRODUCTION}

Let $\partial D$ denote the unit circle of the complex plane. Let

$$
\|P\|:=\max _{z \in \partial D}|P(z)| .
$$

A classical result of Erdős and Turán [3] is the following.

Key words and phrases. polynomials, distribution of zeros, Erdős-Turán Theorem.

2000 Mathematics Subject Classifications: Primary: 41A17 
Theorem (Erdös-Turán). If the zeros of

$$
P(z):=\sum_{j=0}^{n} a_{j} z^{j}, \quad a_{j} \in \mathbb{C}, \quad a_{0} a_{n} \neq 0
$$

are denoted by

$$
z_{j}=r_{j} \exp \left(i \varphi_{j}\right), \quad r_{j}>0, \quad \varphi_{j} \in[0,2 \pi), \quad j=1,2, \cdots, n,
$$

then for every $0 \leq \alpha<\beta \leq 2 \pi$ we have

$$
\left|\sum_{j \in I(\alpha, \beta)} 1-\frac{\beta-\alpha}{2 \pi} n\right|<16 \sqrt{n \log R},
$$

where

$$
R:=\left|a_{0} a_{n}\right|^{-1 / 2}\|P\|
$$

and

$$
I(\alpha, \beta):=\left\{j: \alpha \leq \varphi_{j} \leq \beta\right\} .
$$

The review [6] written by V. Totik on the recent book [1] of V.V. Andrievskii and H-P. Blatt shows the central role of the Erdős-Turán Theorem in certain types of investigations.

Note that some books quote this result with

$$
R:=\left|a_{0} a_{n}\right|^{-1 / 2}\left(\left|a_{0}\right|+\left|a_{1}\right|+\cdots+\left|a_{n}\right|\right)
$$

in place of $R:=\left|a_{0} a_{n}\right|^{-1 / 2}\|P\|$. In fact, the weaker result is an obvious corollary of the stronger one by observing that $\|P\| \leq\left|a_{0}\right|+\left|a_{1}\right|+\cdots+\left|a_{n}\right|$.

\section{New Result}

In this note we offer a subtle "one-sided" improvement of the above Erdős-Turán Theorem.

Theorem 1.1. If the zeros of

$$
P(z):=\sum_{j=0}^{n} a_{j} z^{j}, \quad a_{j} \in \mathbb{C}, \quad a_{0} a_{n} \neq 0,
$$

are denoted by

$$
z_{j}=r_{j} \exp \left(i \varphi_{j}\right), \quad r_{j}>0, \quad \varphi_{j} \in[0,2 \pi), \quad j=1,2, \cdots, n,
$$

then for every $0 \leq \alpha<\beta \leq 2 \pi$ we have

$$
\sum_{j \in I_{1}(\alpha, \beta)} 1-\frac{\beta-\alpha}{2 \pi} n \leq 16 \sqrt{n \log R_{1}},
$$


and

$$
\sum_{j \in I_{2}(\alpha, \beta)} 1-\frac{\beta-\alpha}{2 \pi} n \leq 16 \sqrt{n \log R_{2}}
$$

where

$$
R_{1}:=\left|a_{n}\right|^{-1}\|P\|, \quad R_{2}:=\left|a_{0}\right|^{-1}\|P\|,
$$

and

$$
I_{1}(\alpha, \beta):=\left\{j: \alpha \leq \varphi_{j} \leq \beta, r_{j} \geq 1\right\}, \quad I_{2}(\alpha, \beta):=\left\{j: \alpha \leq \varphi_{j} \leq \beta, r_{j} \leq 1\right\} .
$$

This result is closely related to a recent paper of V. Totik and P. Varjú [7]. In fact, it may as well be derived from part (ii) of Theorem 1 in [7]. However, here we do not rely on this recent result. Our approach is based on the interesting observation that the Erdős-Turán Theorem above improves itself.

Proof. It is sufficient to prove only the first inequality for

$$
P(z):=\sum_{j=0}^{n} a_{j} z^{j}, \quad a_{j} \in \mathbb{C}, \quad a_{0} a_{n} \neq 0 .
$$

If we apply it to

$$
P^{*}(z):=z^{n} \overline{P(1 / \bar{z})}=\sum_{j=0}^{n} \bar{a}_{n-j} z^{j},
$$

we obtain the second inequality of the theorem. Without loss of generality we may also assume that $a_{n}=1$. Let

$$
k:=\sum_{j \in I_{1}(\alpha, \beta)} 1-\frac{\beta-\alpha}{2 \pi} n .
$$

Without loss of generality we may assume that $r_{j}=1$ for each $j \in I_{1}(\alpha, \beta)$. Indeed, if we replace each $z_{j}=r_{j} \exp \left(i \varphi_{j}\right)$ with $r_{j}>1$ with $\widetilde{z}_{j}:=\exp \left(i \varphi_{j}\right)$, and introduce

$$
\widetilde{P}(z)=\prod_{j \in I_{2}(\alpha, \beta)}\left(z-z_{j}\right) \prod_{j \in I_{1}(\alpha, \beta)}\left(z-\widetilde{z}_{j}\right)=\sum_{j=0}^{n} \widetilde{a}_{j} z^{j}
$$

then the polynomial $\widetilde{P}$ is still monic and $\|\widetilde{P}\| \leq\|P\|$. Without loss of generality we may also assume that $k \geq 0$, otherwise there is nothing to prove. We need to prove that

$$
\|P\| \geq \exp \left(\frac{k^{2}}{256 n}\right) .
$$

If $\left|a_{0}\right| \geq 1$, then the result follows from the Erdős -Turán Theorem. Suppose $\left|a_{0}\right|<1$. Let $m$ be a nonnegative integer. Let $S:=P^{m}$. Since each zero of $P$ with multiplicity $u$ on the unit circle is a zero of $S+S^{*}$ with multiplicity at least $m u$, the Erdős-Turán Theorem gives

$$
\left\|S+S^{*}\right\|>\left|1+a_{0}^{m}\right| \exp \left(\frac{(m k)^{2}}{256 m n}\right),
$$


hence

$$
2\|P\|^{m}>\left|1+a_{0}^{m}\right| \exp \left(\frac{m k^{2}}{256 n}\right) .
$$

Therefore

$$
\|P\| \geq\left.\left. 2^{-1 / m}|1-| a_{0}\right|^{m}\right|^{1 / m} \exp \left(\frac{k^{2}}{256 n}\right) \geq\left. 2^{-1 / m}|1-| a_{0}\right|^{m} \mid \exp \left(\frac{k^{2}}{256 n}\right)
$$

follows. Now let $m$ tend to $\infty$. We obtain

$$
\|P\| \geq \exp \left(\frac{k^{2}}{256 n}\right)
$$

and the theorem follows.

As a corollary we obtain a recent result of V. Totik and P. Varjú [7].

Corollary 1.2. If the modulus of a monic polynomial $P$ of degree $n$ (with complex coefficients) on the unit circle of the complex plane is at most $1+o(1)$ uniformly, then the multiplicity of each zero of $P$ on the unit circle is o( $\left.n^{1 / 2}\right)$.

Actually we get this stronger form of the Totik-Varjú result:

\section{Corollary 1.3.}

(i) If the modulus of a monic polynomial $P$ of degree $n$ (with complex coefficients) on the unit circle of the complex plane is at most $1+o(1)$ uniformly, then the multiplicity of each zero of $P$ outside the open unit disk is o $\left(n^{1 / 2}\right)$.

(ii) Equivalently, if a complex polynomial $P$ of degree $n$ and constant term 1 has modulus at most $1+o(1)$ uniformly on the unit circle, then the multiplicity of each zero of $P$ in the closed unit disk is o $\left(n^{1 / 2}\right)$.

Proof. Suppose $P$ is a monic polynomial of degree $n$ with $k$ zeros at a point $z_{0}$ outside the open unit disk. Then Theorem 1.1 implies

$$
\|P\| \geq \exp \left(\frac{k^{2}}{256 n}\right)
$$

and the corollary follows.

We note that Corollary 1.3 extends to generalized polynomials of the form

$$
f(z)=\prod_{j=1}^{k}\left(z-z_{j}\right)^{r_{j}}, \quad z_{j} \in \mathbb{C}, r_{j}>0
$$

where $N:=\sum_{j=1}^{k} r_{j}$ is the degree of the generalized polynomial. 


\section{REMARKS}

A construction of G. Halász [4] shows that for every $k \in \mathbb{N}$, there exists a polynomial $h$ of degree $k$ (with real coefficients) such that

$$
h(0)=1, \quad h(1)=0, \quad \text { and } \quad|h(z)|<\exp \left(\frac{2}{k}\right) \quad \text { for all } \quad z \in \partial D .
$$

(See also [5] concerning the exact constant.) This implies the following observation.

Remark 2.1. $o\left(n^{1 / 2}\right)$ in Corollary 1.2 cannot be improved.

Proof. Indeed, let the polynomial $h$ of degree $k$ be picked by the above lemma. Then $H:=h^{m}$ is a polynomial of degree $k m, H(0)=1$, and $H$ has a zero at 1 with multiplicity at least $m$. Also $|H(z)|<\exp (2 m / k)$ for all $z \in \partial D$. Now let $Q$ be defined by $Q(z):=z^{k m} H(1 / z)$. Then $Q$ is a monic polynomial of degree $k m$ and $Q$ has a zero at 1 with multiplicity at least $m$. Also $|Q(z)|<\exp (2 m / k)$ for all $z \in \partial D$. Now choose $m=o(k)$.

Remark 2.2. A result closely related to Theorem 1.1 is the theorem below. See Theorem 4.1 in [2]. However, Theorem 1.1 does not follow from this.

Theorem (Borwein-Erdélyi-Kós). There is an absolute constant $c>0$ such that every polynomial $p$ of the form

$$
p(x)=\sum_{j=0}^{n} a_{j} x^{j}, \quad\left|a_{j}\right| \leq 1, \quad\left|a_{0}\right|=1, \quad a_{j} \in \mathbb{C},
$$

has at most $c \sqrt{n}$ zeros in $[-1,1]$.

There is an absolute constant $c>0$ such that every polynomial $p$ of the form

$$
p(x)=\sum_{j=0}^{n} a_{j} x^{j}, \quad\left|a_{j}\right| \leq 1, \quad\left|a_{n}\right|=1, \quad a_{j} \in \mathbb{C},
$$

has at most $c \sqrt{n}$ zeros in $\mathbb{R} \backslash(-1,1)$.

There is an absolute constant $c>0$ such that every polynomial $p$ of the form

$$
p(x)=\sum_{j=0}^{n} a_{j} x^{j}, \quad\left|a_{j}\right| \leq 1, \quad\left|a_{0}\right|=\left|a_{n}\right|=1, \quad a_{j} \in \mathbb{C},
$$

has at most $c \sqrt{n}$ real zeros.

\section{REFERENCES}

1. V.V. Andrievskii and H-P. Blatt, Discrepancy of Signed Measures and Polynomial Approximation, Springer, New York, 2002. 
2. P. Borwein, T. Erdélyi, and G. Kós, Littlewood-type problems on [0,1], Proc. London Math. Soc. (3) 79 (1999), 22-46.

3. P. Erdős and P. Turán, On the distribution of roots of polynomials, Ann. Math. (1950), 105-119.

4. G. Halász, On the first and second main theorem in Turán's theory of power sums, Studies in Pure Mathematics, To the Memory of Paul Turán (P. Erdős, ed.), Birkhäuser Verlag, Basel, 1983, pp. 259-269.

5. M. Lachance, E.B. Saff, and R. Varga, Inequalities for polynomials with a prescribed zero, Math. Z. 168 (1979), 105-116.

6. V. Totik, Review on the book "Discrepancy of Signed Measures and Polynomial Approximation", Bull. London Math. Soc. 35 (2003), 284-287.

7. V. Totik and P. Varjú, Polynomials with prescribed zeros and small norm, Acta Sci. Math. (Szeged) (to appear). 